\title{
Functionalized carbon nanomaterials: exploring the interactions with Caco-2 cells for potential oral drug delivery
}

This article was published in the following Dove Press journal:

International Journal of Nanomedicine

7 October 2011

Number of times this article has been viewed

\section{Jurja C Coyuco \\ Yuanjie Liu \\ Bee-Jen Tan \\ Gigi NC Chiu}

Department of Pharmacy, Faculty of Science, National University

of Singapore, Singapore

Video abstract

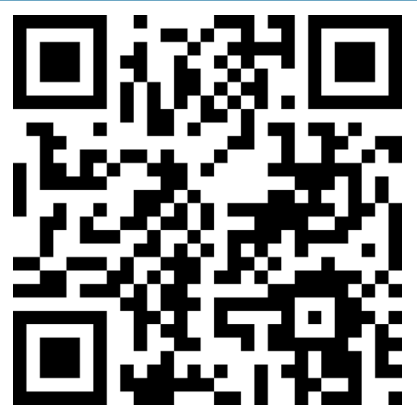

Point your SmartPhone at the code above. If you have a QR code reader the video abstract will appear. Or use: http://dvpr.es/ijncoyuco

Correspondence: Gigi NC Chiu Department of Pharmacy, Faculty of Science, National University of Singapore, 18 Science Drive 4, Singapore 117543

Tel +65 65I65536

Fax +6567791554

Email phacncg@nus.edu.sg
Abstract: Although carbon nanomaterials (CNMs) have been increasingly studied for their biomedical applications, there is limited research on these novel materials for oral drug delivery. As such, this study aimed to explore the potential of CNMs in oral drug delivery, and the objectives were to evaluate CNM cytotoxicity and their abilities to modulate paracellular transport and the P-glycoprotein (P-gp) efflux pump. Three types of functionalized CNMs were studied, including polyhydroxy small-gap fullerenes (OH-fullerenes), carboxylic acid functionalized single-walled carbon nanotubes ( $f$ SWCNT-COOH) and poly(ethylene glycol) functionalized single-walled carbon nanotubes ( $f$ SWCNT-PEG), using the well-established Caco-2 cell monolayer to represent the intestinal epithelium. All three CNMs had minimum cytotoxicity on Caco-2 cells, as demonstrated through lactose dehydrogenase release and 3-(4,5-dimethyliazol-2-yl)-2,5-diphenyltetrazolium bromide (MTT) assays. Of the three CNMs, $f$ SWCNT-COOH significantly reduced transepithelial electrical resistance and enhanced transport of Lucifer Yellow across the Caco-2 monolayer. Confocal fluorescence microscopy showed that $f$ SWCNT-COOH treated cells had the highest perturbation in the distribution of ZO-1, a protein marker of tight junction, suggesting that $f$ SWCNT-COOH could enhance paracellular permeability via disruption of tight junctions. This modulating effect of $f$ SWCNT-COOH can be reversed over time. Furthermore, cellular accumulation of the P-gp substrate, rhodamine-123, was significantly increased in cells treated with $f$ SWCNT-COOH, suggestive of P-gp inhibition. Of note, $f$ SWCNT-PEG could increase rhodamine-123 accumulation without modifying the tight junction. Collectively, these results suggest that the functionalized CNMs could be useful as modulators for oral drug delivery, and the differential effects on the intestinal epithelium imparted by different types of CNMs would create unique opportunities for drug-specific oral delivery applications.

Keywords: fullerenes, carbon nanotubes, functionalization, paracellular transport, P-glycoprotein

\section{Introduction}

Carbon-based nanomaterials (CNMs) have rapidly emerged as important components in a wide variety of applications such as electronics, computer, aerospace, architecture, and potentially medicine in drug delivery and prosthetics. ${ }^{1-4}$ Owing to their unique electrical, mechanical, and thermal properties, ${ }^{2} \mathrm{CNMs}$ have attracted intense academic and industrial interest ever since their first description. ${ }^{5,6}$ The most commonly investigated CNMs include fullerenes, single-walled and multi-walled carbon nanotubes. Recently, graphene and its derivatives have emerged as yet another type of CNM of immense interest in biomedical applications such as drug delivery. ${ }^{7-9}$ However, before the potential for biomedical application can be realized, the toxicity profiles of CNMs 
must be characterized to the satisfaction of regulatory bodies so that CNMs can be incorporated as safe components in medicinal products.

Most of the toxicological data have been obtained using in vitro human cell models such as dermal fibroblasts, ${ }^{10}$ bronchial epithelial cells, ${ }^{11}$ macrophages, ${ }^{12}$ and embryonic kidney (HEK293) cells. ${ }^{13}$ Animal models have also been used to elucidate the potential toxicity of CNMs, with rodents being the most commonly used. From these studies, the toxicity of CNMs can be linked to a number of factors including metal catalyst contamination, particle size or length, surface area and functionalization, and aggregation state as well as route of exposure..$^{1,3,14,15}$ Route of exposure or administration is an important factor in governing the type of toxicity that is ultimately manifested in the exposed animal or human. Studies investigating this factor would shed light on the potential of CNMs for biomedical applications such as drug delivery systems. Various routes of administration have been explored with CNMs including intravenous, intraperitoneal, oral, and intrascrotal. Most data have been collected on the intraperitoneal route and have demonstrated granuloma formation resulting from carbon nanotube exposure. ${ }^{16-18}$ While the intraperitoneal route is commonly used in rodents because of ease of administration, its use in humans is mostly limited to peritoneal dialysis and administration of chemotherapy for diseases of the peritoneal cavity. Because CNMs are water-insoluble, dosing animals intravenously might give rise to mechanical blockage of blood vessels, ${ }^{16}$ thus limiting the potential of CNMs as delivery vehicles for intravenous application that necessitates appropriate functionalization to improve biocompatibility. ${ }^{14,15}$ Considering marketed drug products, oral delivery is the most common and most desirable route of administration. Despite the limited and fragmentary toxicological information gathered from oral CNM exposure, a few recent studies have shown that these nanomaterials when given orally do not give rise to the toxic symptoms produced by intraperitoneal exposure. ${ }^{16,19-21}$ These positive studies have prompted us to investigate the potential of CNMs as oral drug delivery vehicles.

In particular, we have explored the interactions of three functionalized CNMs, including: polyhydroxylated fullerenes (OH-fullerenes); single-walled carbon nanotubes functionalized with carboxylic acid ( $f$ SWCNT-COOH); and single-walled carbon nanotubes functionalized with polyethylene glycol ( $f$ SWCNT-PEG), with Caco-2 cell monolayer. These functionalized materials were selected based on their improved dispersibility in aqueous media, which could lend better biocompatibility. 14,15,22 They have also been intensively studied, thus allowing comparison to existing literature. The Caco-2 cell monolayer has been well established as an in vitro model for the study of oral drug absorption. ${ }^{23}$ The differentiated cell monolayer has tight junctions and drug efflux transporters and thus resembles the normal intestinal epithelium as a drug absorption barrier. ${ }^{24,25} \mathrm{~A}$ desirable oral drug delivery system should have the ability to modulate the intestinal absorptive barrier so as to transport drugs across and promote absorption, in addition to being minimally toxic to the intestinal barrier. The current study is the first to report on the ability of functionalized CNMs to modulate drug transport across the Caco-2 intestinal epithelium.

\section{Materials and methods Materials}

The CNMs used in this study were all purchased from SigmaAldrich Inc (Singapore), including the hydrated, polyhydroxy small gap fullerenes $\left(\mathrm{C}_{120} \mathrm{O}_{30}(\mathrm{OH})_{30} \cdot 30 \mathrm{H}_{2} \mathrm{O} \cdot 25 \mathrm{Na}^{+}\right.$, cat \# 707481 ), the $f$ SWCNT-COOH nanotubes (with extent of labeling of 2.0 atom $\%$ carboxylic groups, cat \# 652490), and the $f$ SWCNT-PEG nanotubes (with $\mathrm{PEG}_{600}$ :SWCNT weight ratio of 20:80, cat \# 652474). Both SWCNT were produced by electric arc discharge method as described by the supplier. Caco-2 cell line was purchased from the American Type Culture Collection (Manassas, VA), while the cell culture media and supplements were purchased from MP Biomedicals (Illkirch, France).

\section{Dispersion of CNMs in aqueous media}

The three CNMs were used as received without further purification steps. The CNMs were dispersed in Hanks's balanced salt solution (HBSS) containing $10 \mathrm{mM}$ 4-(2-hydroxyethyl)1-piperazineethanesulfonic acid (HEPES) by sonication for 1 hour using a Branson 2510 bath sonicator (Danbury, CT) to produce a fine suspension at a concentration of $1 \mathrm{mg} / \mathrm{mL}$ for subsequent experimentation.

\section{Characterization of CNMs}

Scanning electron microscopy (SEM) of the powder form of the three CNMs was performed with a JEOL JSM-6701F (JEOL, Tokyo, Japan) field emission scanning electron microscope. Transmission electron microscopy (TEM) of the aqueous-dispersed CNMs was performed with a JEOL JEM-2010F field emission transmission electron microscope (JEOL, Tokyo, Japan). Metal content (Co, Ni, and Fe) was determined by inductively coupled plasma optical emission 
spectrometry (ICP-OES) analysis with Dual-view Optima 5300 DV ICP-OES system (Perkin Elmer Inc, Shelton, CT).

\section{Cell culture}

The Caco- 2 cell line was used within 20 passages from thawing. Cells were grown in a humidified atmosphere of $5 \% \mathrm{CO}_{2}$ at $37^{\circ} \mathrm{C}$ and maintained in Eagle's minimal essential medium (EMEM) supplemented with $20 \%$ fetal bovine serum (FBS), 1\% L-glutamine, 100 units $/ \mathrm{mL}$ penicillin, and $100 \mu \mathrm{g} / \mathrm{mL}$ streptomycin. Stock cultures were grown in T-75 $\mathrm{cm}^{2}$ flasks, with medium renewal performed every 3-4 days. Subculturing of cells was done when the culture reached $80 \%-90 \%$ confluence.

\section{Cell cytotoxicity and viability assay}

For the evaluation of cytotoxicity, the lactose dehydrogenase (LDH) release assay was used. Briefly, Caco-2 cells were seeded in 96-well plates at a density of $1.33 \times 10^{4}$ cells/well and incubated for 21 days under the same culture conditions as described in the previous section. After 21 days, the medium was aspirated and replaced with serum-supplemented medium containing serial dilutions of the three CNMs. Triton-X at $0.05 \%$ was used as the control for maximum LDH release. The cells were exposed to the CNMs for 24 hours at $37^{\circ} \mathrm{C}$, after which the culture medium was collected for LDH detection using Cytotox-One Homogenous Membrane Integrity Assay Kit (Promega Corporation, Madison, WI) following manufacturer's instructions. Fluorescence was measured using a Tecan SpectraFluor Plus plate reader (Tecan Group Ltd, Mannedorf, Switzerland) with excitation wavelength of $560 \mathrm{~nm}$ and emission wavelength of $590 \mathrm{~nm}$. Cytotoxicity was calculated as follows:

$$
\text { Cytotoxicity }(\%)=\frac{F_{\text {test }}-F_{\text {background }}}{F_{\text {triton-X }}-F_{\text {background }}} \times 100 \%
$$

where $F_{\text {test }}, F_{\text {triton-X } X}$ and $F_{\text {background }}$ represent the fluorescence readings from the test condition (CNM or vehicle alone), triton-X $0.05 \%$, and background.

For the cell viability assay, treatment groups and conditions were similar to those for the LDH release assay, except that 3-(4,5-dimethyliazol-2-yl)-2,5-diphenyltetrazolium bromide (MTT) was added to each well after medium removal and phosphate buffered saline (PBS) rinsing to remove residual CNM. Following 4 hours of incubation at $37^{\circ} \mathrm{C}$, the well content was aspirated and $150 \mu \mathrm{L}$ dimethylsulfoxide (DMSO) was added to dissolve formazan crystals. Absorbance at
$570 \mathrm{~nm}$ was measured with a Tecan SpectraFluorPlus reader. Cell viability was calculated as follows:

Viability $(\%)=\frac{A b s_{\text {test }}-A b s_{\text {background }}}{A b s_{\text {vehicle control }}-A b s_{\text {background }}} \times 100 \%$

where $A b s_{\text {test }}, A b s_{\text {background }}, A b s_{\text {vehicle control }}$ represent the absorbance readings from the CNM-treated wells, the mediumonly wells, and the vehicle control wells, respectively.

\section{Transepithelial electrical resistance (TEER) measurement in Caco-2 cells}

Cells were seeded at a density of $1.2 \times 10^{4}$ cells/well in collagen-coated filter membrane polycarbonate transwell inserts ( $12 \mathrm{~mm}, 1 \mu \mathrm{m}$ pore size, $0.3 \mathrm{~cm}^{2}$ growth area) obtained from BD Biocoat (Erembodegem, Belgium) and incubated at $37^{\circ} \mathrm{C}$ for 21 days under the same culture conditions described in the previous section. The volumes of media were $0.2 \mathrm{~mL}$ and $0.5 \mathrm{~mL}$ on the apical and basolateral side, respectively. On day 21, the baseline transepithelial electrical resistance (TEER) values of Caco-2 cells were measured using Millicell ERS-2 Epithelial Volt-Ohm Meter to ensure cell monolayer integrity. Inserts that records $>600 \Omega \mathrm{cm}^{2}$, which indicated good monolayer integrity, were subjected to experimentation. Media in both sides of the chambers were aspirated, and the chamber was washed twice with HBSS. The Caco-2 cells in the inserts were exposed to either HBSS buffer or CNM at $1 \mathrm{mg} / \mathrm{mL}$ in HBSS for 24 hours at $37^{\circ} \mathrm{C}$. The TEER values of these cells after treatment were recorded. Percent change in TEER after CNM exposure was calculated as follows:

$$
\operatorname{TEER}(\%)=\frac{R_{\text {test }}-R_{\text {background }}}{R_{\text {vehicle control }}-R_{\text {background }}} \times 100 \%
$$

where $R_{\text {test }}, R_{\text {background }}, R_{\text {vehicle control }}$ represent the TEER readings from the CNM-treated wells, the no-cell control wells, and the vehicle control wells, respectively.

\section{Transport of Lucifer Yellow (LY) across Caco-2 monolayer}

Similar to the conditions for TEER measurements, Caco-2 cells were grown in the transwell inserts for 21 days before experimentation. The cells were treated with either HBSS buffer or $1 \mathrm{mg} / \mathrm{mL}$ of the CNM for 24 hours. At the end of CNM exposure, cells were washed three times with HBSS. Lucifer Yellow (100 $\mu \mathrm{M}$ in HBSS) was added to the apical chamber at a level of $200 \mu \mathrm{L}$, and $500 \mu \mathrm{L}$ HBSS was 
added to the basolateral chamber. At 1, 2, 4, and 24 hours, $250 \mu \mathrm{L}$ of sample from the basolateral chamber was removed and replaced with the same volume of fresh HBSS buffer. Samples collected from the basolateral wells were then quantified using Tecan SpectraFluorPlus reader with excitation/emission wavelength of $430 / 540 \mathrm{~nm}$.

\section{Tight junction staining with anti-ZO-I antibody}

Caco-2 cells were seeded in 4-well Lab-tek chamber glass slides from Nalge Nunc Inc (Naperville, IL) at a density of $7.210^{4}$ cells/well and cultured for 21 days before experimentation. Cells were exposed to $500 \mu \mathrm{L}$ HBSS (vehicle control), $0.05 \%$ triton-X, or $1 \mathrm{mg} / \mathrm{mL} \mathrm{CNM}$ for 4 and 24 hours at $37^{\circ} \mathrm{C}$. After incubation, cells were washed three times with ice-cold PBS, and fixed with ice-cold methanol for 10 minutes. Subsequently, cells were rinsed with ice-cold PBS and blocked with $1 \%$ BSA in PBS for 30 minutes. Primary antibody (purified mouse anti-human ZO-1 obtained from BD Transduction Laboratories, San Jose, CA) was added at $2 \mu \mathrm{g} / \mathrm{mL}$ and incubated at room temperature for 1 hour. The wells were then washed three times with PBS, with 5 minutes incubation after each wash, before the incubation with the secondary antibody (anti-mouse alexa-fluor 568) at $4 \mu \mathrm{g} / \mathrm{mL}$ for 1 hour at room temperature. Finally, cells were washed three times with PBS before viewing under a Carl Zeiss fluorescence confocal microscope using $40 \times$ objective (Carl Zeiss, Jena, Germany).

\section{Rhodamine- 123 accumulation in Caco- 2 cells}

Cells were grown in 24-well culture slides at a density of $8 \times 10^{4}$ cells/well and incubated for 21 days before experimentation. Cells were exposed to either serial dilutions of $\mathrm{CNM}$ or HBSS for 90 minutes at $37^{\circ} \mathrm{C}$, and were washed twice with HBSS at the end of incubation. Rhodamine-123 (R-123, at $5 \mu \mathrm{M}$ ) was subsequently added to the cells and incubated for 90 minutes at $37^{\circ} \mathrm{C}$. Cyclosporin $\mathrm{A}$, an established P-glycoprotein (P-gp) inhibitor, was used as a positive control. After incubation, cells were washed with PBS and lysed with $1 \%$ triton-X to release the intracellular R-123. After centrifuging at 13,000 rpm for 10 minutes to remove cell debris, cell lysates were transferred to a 96-well plate for measuring fluorescence intensity of R-123 using a Tecan SpectraFluorPlus reader with excitation/emission wavelength of $485 / 530 \mathrm{~nm}$. The amount of R-123 accumulated was normalized to the protein content of the cells determined using the Bio-Rad protein assay kit (Bio-Rad, Hercules, CA) as described by the manufacturer's instructions.

\section{Statistical analysis}

Where appropriate, results were expressed as mean \pm SEM of at least three independent experiments. Statistical significance was assessed using one-way ANOVA followed by Fisher's least significant difference (LSD) test for multiple comparisons. A $P$ value of $<0.05$ was considered statistically significant. Statistical analyses were carried out using SPSS software (v 19.0; IBM, Armonk, NY).

\section{Results}

\section{Characterization of the functionalized CNMs}

The three functionalized CNMs were used as received without further purification steps. Table 1 summarizes the characteristics of the three functionalized CNMs. Parameters including diameter, bundle dimension for the $f$ SWCNTs, and carbonaceous purity were provided by the supplier. Common metal impurities, including cobalt, nickel, and iron, which are important in inducing toxicity to cells, were determined by ICP-OES. In general, the three metals were present in $<1 \%$ by weight in the three CNM products. Figure 1 shows the SEM images of the CNM powder as received and the TEM images of the CNMs dispersed in HBSS. From these images, the three CNMs were in an aggregated state although they have been functionalized with hydrophilic groups to achieve better dispersibility than the non-functionalized, pristine forms.

Table I Characterization of the functionalized carbon-based nanomaterials

\begin{tabular}{|c|c|c|c|}
\hline & OH-fullerenes & fSWCNT-COOH & fSWCNT-PEG \\
\hline Diameter $(\mathrm{nm})^{\mathrm{a}}$ & 0.7 & $1.4 \pm 0.1$ & $1.4 \pm 0.1$ \\
\hline Bundle dimension & NA & $4-5 \mathrm{~nm} \times 0.5-\mathrm{I} .5 \mu \mathrm{m}$ & $4-5 \mathrm{~nm} \times 0.5-0.6 \mu \mathrm{m}$ \\
\hline $\mathrm{Co} / \mathrm{Ni} / \mathrm{Fe}$ content $(w t \%)^{\mathrm{b}}$ & Und/Und/0.39\% & Und $/ 0.36 \% / 0.19 \%$ & Und $/ 0.26 \% / 0.21 \%$ \\
\hline Carbonaceous purity & ND & $>90 \%$ & $>80 \%$ \\
\hline
\end{tabular}

Notes: aParameters including diameter, bundle dimension and carbonaceous purity were provided by the source supplier (Sigma-Aldrich, St Louis, MO); ${ }^{\text {as }}$ determined by elemental analysis (Dual-view Optima 5300 DV ICP-OES system).

Abbreviations: NA, not applicable; ND, no data; Und, undetected; OH-fullerenes, polyhydroxylated fullerenes; fSWCNT-COOH, single-walled carbon nanotubes functionalized with carboxylic acid; fSWCNT-PEG, single-walled carbon nanotubes functionalized with polyethylene glycol. 


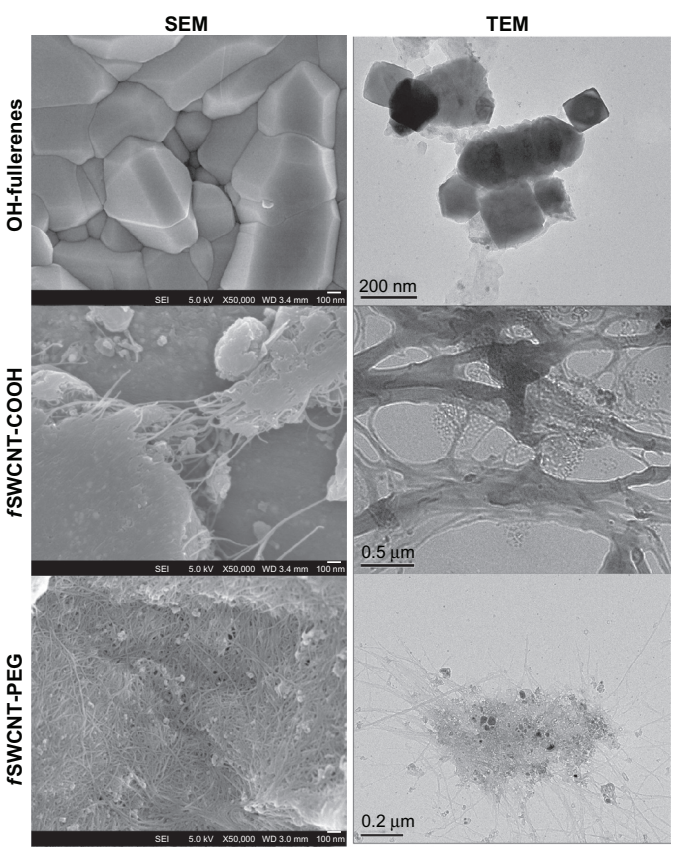

Figure I SEM and TEM of OH-fullerenes, fSWCNT-COOH, and fSWCNT-PEG. Abbreviations: SEM, scanning electron microscopy; TEM, transmission electron microscopy; $\mathrm{OH}$-fullerenes, polyhydroxylated fullerenes; fSWCNT-COOH, carboxylic acid functionalized single-walled carbon nanotubes; fSWCNT-PEG, poly(ethylene glycol) functionalized single-walled carbon nanotubes.

\section{Cytotoxicity of the functionalized CNMs in Caco-2 cell monolayer}

The cytotoxicity of the three functionalized CNMs in Caco-2 cells was determined by the LDH release assay, which is an established cytotoxicity assay that probes plasma membrane integrity. To determine whether the functionalized CNMs could interfere with the assay, Caco-2 cells were lysed with $1 \%$ triton-X to yield cell lysate with which LDH activity was measured in the presence and absence of the CNMs. No significant difference in the generation of the fluorescent resorufin product was observed between these two testing conditions (data not shown). As shown in Figure 2,

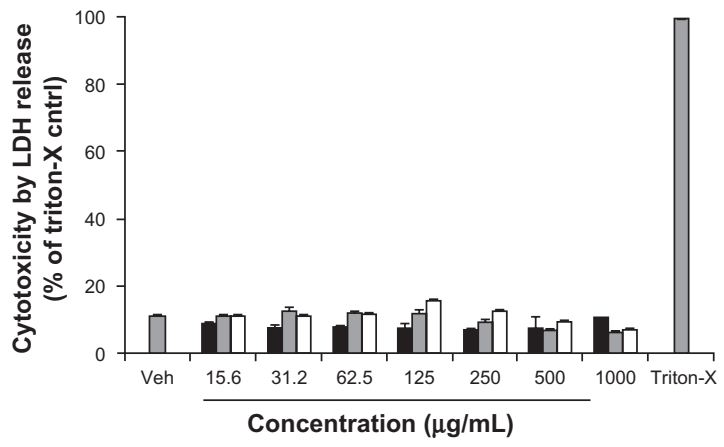

Figure 2 Cytotoxicity of OH-fullerenes ( $\square)$, fSWCNT-COOH ( $\square$ ), and fSWCNTPEG ( $\square$ ) after 24 hours of treatment.

Abbreviations: $\mathrm{LDH}$, lactose dehydrogenase; $\mathrm{OH}$-fullerenes, polyhydroxylated fullerenes; fSWCNT-COOH, carboxylic acid functionalized single-walled carbon nanotubes; fSWCNT-PEG, poly(ethylene glycol) functionalized single-walled carbon nanotubes. the toxicity of Caco-2 cells exposed to $\mathrm{OH}$-fullerenes, $f$ SWCNT-COOH, and $f$ SWCNT-PEG for 24 hours was minimal over the concentration range of $15.6-1000 \mu \mathrm{g} / \mathrm{mL}$, and no significant difference was observed when the cytotoxicity of the three CNM groups was compared to the vehicle control $(P>0.05)$.

The MTT assay which measures the mitochondrial activity in living cells is an established and popular assay to determine cytotoxicity. Of note, the reliability of the MTT assay in determining the cytotoxicity of non-functionalized, pristine SWCNT has been scrutinized, ${ }^{26,27}$ yet studies evaluating potential interference from functionalized CNMs in MTT assays are lacking. In view of the limited data available, the MTT assay was performed on the Caco-2 cells treated with the CNMs, in a way similar to the LDH release assay, in the concentration range of $15.6-1000 \mu \mathrm{g} / \mathrm{mL}$. Our results indicated that all three functionalized CNMs tested up to the highest concentration of $1000 \mu \mathrm{g} / \mathrm{mL}$, did not significantly reduce the viability of Caco-2 cells as compared to vehicle control (Supplementary Figure 1). This finding corroborates with the LDH release assay that the three CNMs did not induce significant damage or cytotoxicity in Caco-2 cells.

As exposure of 24 hours may not be sufficient to demonstrate that the CNMs are safe to cells, further studies involving longer exposure time were conducted using the LDH release assay (Supplementary Figure 2). After 72 hours of exposure, $\mathrm{OH}$-fullerenes and $f$ SWCNT-COOH did not have increased cytotoxicity, but a slight increase in the cytotoxicity of fSWCNT-PEG from 10\% (24 hours treatment) to 30\%-35\% (72 hours treatment) was observed, which is somewhat surprising given that $\mathrm{PEG}$ functionalization of $\mathrm{CNM}$ yields derivatives of lower toxicity. Further investigation comparing the intracellular fate of these functionalized CNMs in Caco-2 cells would shed light on the differential cytotoxicity upon longer exposure to the CNMs. However, compared to other well-accepted PEG-derivatized modulators such as Pluronic-P85 and vitamin E-TPGS 1000, the LDH release induced by $f$ SWCNT-PEG is comparable. This is based on published reports that used Caco-2 cells as the model with cytotoxicity measured by LDH release, ${ }^{28,29}$ and showed that the cytotoxicity of $1 \mathrm{mg} / \mathrm{mL}$ vitamin E-TPGS 1000 or Pluronic-P85 was 30\%-40\% upon an exposure time of 1.5 and 4 hours, respectively.

After evaluating the cytotoxic potential of the functionalized CNMs in Caco-2 cells, it is of interest to examine if these novel materials could modulate the Caco-2 intestinal barrier function, from which insights into the potential of these CNMs as oral delivery vehicles could be obtained. Barriers to oral drug delivery include primarily the presence of tight 
junctions and the expression of drug efflux pumps in the intestinal epithelium. As such, subsequent evaluations were performed to probe the effects on tight junction integrity and drug efflux pump function as a result of CNM exposure.

\section{Tight junction integrity of Caco-2 cell monolayer after exposure to the functionalized CNMs}

Disruption of the tight junctions in the intestinal epithelium would make drug transport via the paracellular route possible. In this regard, tight junction integrity was examined by both morphological and functional properties, including the staining of ZO-1, a major tight junction-associated protein in Caco- 2 cells, ${ }^{30,31}$ TEER measurements, and transport of LY which is a probe impermeable to Caco-2 monolayer when the tight junction is intact. Caco-2 cells were exposed to $1 \mathrm{mg} / \mathrm{mL}$ OH-fullerenes, $f$ SWCNT-COOH, and $f$ SWCNT-PEG for 4 and 24 hours, and subsequently, ZO-1 was detected by immunofluorescence with confocal microscopy. As seen in Figure 3A, cells treated with vehicle control (HBSS) showed a strong, gasket-like and uniform distribution of ZO-1 (Figure 3A), ${ }^{31}$ while cells treated with triton-X, which served

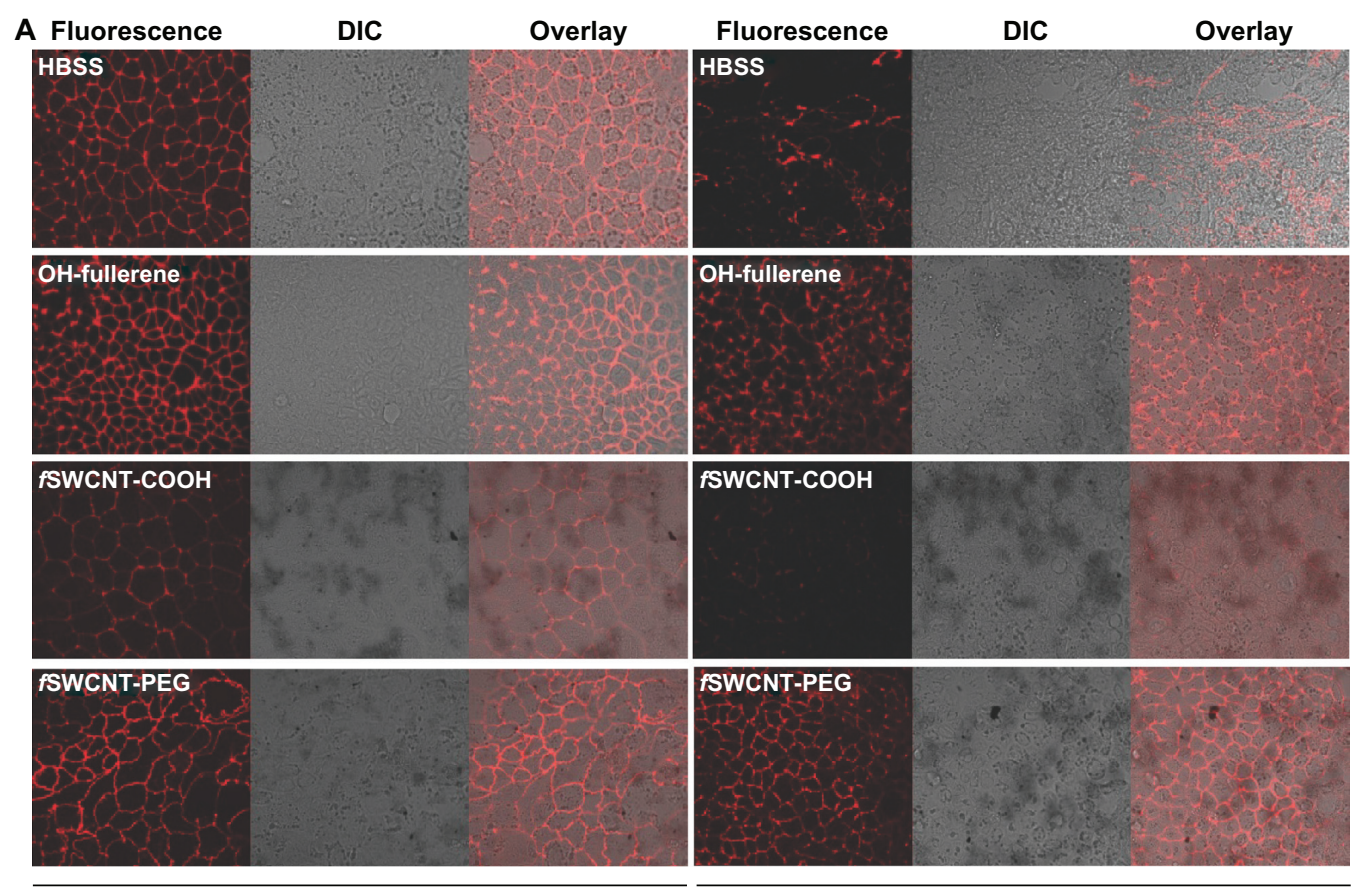

B

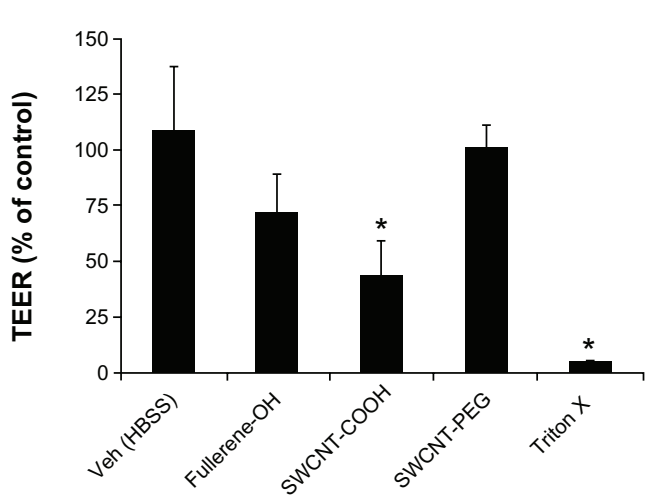

C

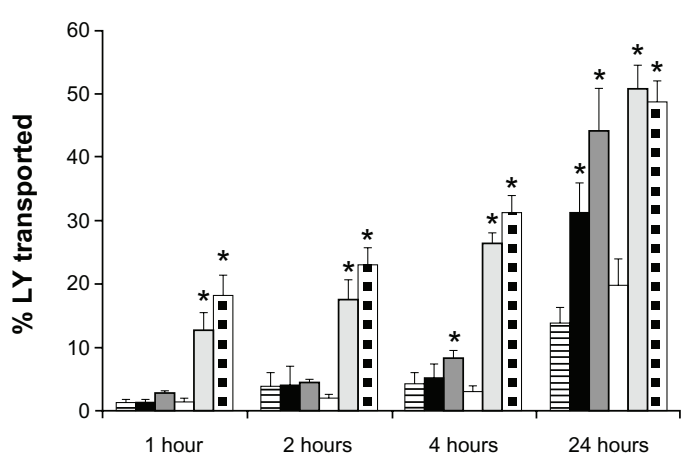

Figure 3 Tight junction modulating effect of OH-fullerenes, fSWCNT-COOH and fSWCNT-PEG in Caco-2 cell monolayer. (A) ZO-I staining upon exposure to the three functionalized CNM for 4 and 24 hours. (B) TEER measurement upon exposure to I mg/mL OH-fullerenes, fSWCNT-COOH or fSWCNT-PFG for 24 hours. (C) Transport of LY over time upon exposure to I mg/mL OH-fullerenes ( $\square$ ), fSWCNT-COOH (dark gray), fSWCNT-PEG ( $\square$ ), with HBSS as negative control (column with horizontal stripes), triton-X (light gray), and cell-free inserts (column with square dots) as positive controls.

Note: Asterisks represent statistical difference as compared to control with $P<0.05$.

Abbreviations: DIC, differential interference contrast; fSWCNT-COOH, carboxylic acid functionalized single-walled carbon nanotubes; fSWCNT-PEG, poly(ethylene glycol) functionalized single-walled carbon nanotubes; LY, lucifer yellow; OH-fullerenes, polyhydroxylated fullerenes. 
as the positive control, showed disrupted and abnormal localization of ZO-1 (Figure 3A). Of the three functionalized CNMs evaluated, only $f$ SWCNT-COOH showed a weakening of ZO-1 immunofluorescence after 4 hours, which was further reduced after 24 hours (Figure 3A). Slight reduction in ZO-1 immunofluorescence could be noticed in cells treated with $\mathrm{OH}$-fullerenes for 24 hours (Figure 3A). ZO-1 immunofluorescence was largely unaffected upon exposure to $f$ SWCNT-PEG for 24 hours (Figure $3 \mathrm{~A}$ ).

In addition to morphological examination, TEER measurement was performed on Caco-2 cells treated with the three functionalized CNMs. A decrease in TEER reflects weakening of the tight junction that results in paracellular transport. ${ }^{32}$ Differentiated Caco-2 monolayers grown in transwell inserts were exposed to $1 \mathrm{mg} / \mathrm{mL}$ of the three functionalized CNMs for 24 hours. As shown in Figure 3B, decreases in TEER could be observed when Caco-2 cells were exposed to the three CNMs; however, only the group treated with $f$ SWCNT$\mathrm{COOH}$ was significantly lower compared to the control $(P<0.05)$. $f$ SWCNT-PEG had the least effect on the TEER of Caco-2 cells. LY transport studies were carried out to further support our TEER observations. The positive control for this study included the cell-free insert and inserts with Caco-2 cells treated with triton-X, and both controls showed significantly higher LY transport across the monolayer throughout the study period (Figure 3C). Of note, LY was added to the transwell inserts after the monolayer was washed to remove residual CNM, to avoid LY binding to residual CNM that might potentially confound the results. As early as 4 hours, Caco-2 cells treated with $f$ SWCNT-COOH showed significantly higher LY transport, an observation that is in line with the ZO-1staining results (Figure 3A). At 24 hours, Caco-2 cells treated with $\mathrm{OH}$-fullerenes and $f$ SWCNT-COOH showed significantly higher LY transport compared to the control, and the extent of LY transport upon $f$ SWCNT-COOH exposure was comparable to that of cell-free control. $f$ SWCNT-PEG exposure did not enhance LY transport over the study period of 24 hours. Taken together, the results from morphological and functional evaluation are in line, and $f$ SWCNT-COOH appears to be the most promising among the three functionalized CNMs in modulating tight junctions.

It is of importance to determine whether the tight junction modulating effect by $f$ SWCNT-COOH is reversible, as irreversibility would represent potential toxicity to the intestinal epithelium and $f$ SWCNT-COOH may not be a good material for oral drug delivery. Therefore, time-course TEER measurements were carried out on Caco-2 monolayers exposed to $f$ SWCNT-COOH and triton-X (as positive control) for 24 hours to examine if initial TEER reduction could be reversed over time. As shown in Figure 4, TEER was gradually increased over time after the removal of $f$ SWCNT-COOH from the culture medium, and could return to approximately $100 \%$ of initial value. In contrast, TEER of Caco- 2 monolayers treated with triton-X remained significantly lower than initial value over the 72-hour study period, indicating the irreversibility of triton-X treatment. Collectively, the results presented in Figures 3 and 4 demonstrated the potential of $f$ SWCNT-COOH in modulating the barrier function of intestinal epithelium by reversibly disrupting tight junctions and promoting paracellular drug transport.

\section{$\mathrm{R}-\mathrm{I} 23$ accumulation studies in Caco- 2 cells}

Another barrier to oral drug delivery is the presence of drug efflux pumps in the intestinal epithelium. It was thus of interest to determine whether the three functionalized CNMs could inhibit the P-gp efflux pump, thereby increasing P-gp drug substrate accumulation in Caco-2 cells. Various concentrations $(1000,500,250,125,62.5 \mu \mathrm{g} / \mathrm{mL})$ of the three functionalized CNMs were used to treat Caco-2 cells, and the cells were washed before the addition of the P-gp substrate R-123 to prevent any adsorption between CNMs and R-123. From Figure 5, a trend whereby an increase in R-123 accumulation in CNM-treated Caco-2 cells could be observed. Of note, $f$ SWCNT-COOH at 500 and $1000 \mu \mathrm{g} / \mathrm{mL}$ and $f$ SWCNT-PEG at $1000 \mu \mathrm{g} / \mathrm{mL}$ significantly increased R-123 accumulation by approximately 2.5 -fold over control $(P<0.05)$, and the fold increase was higher as compared to

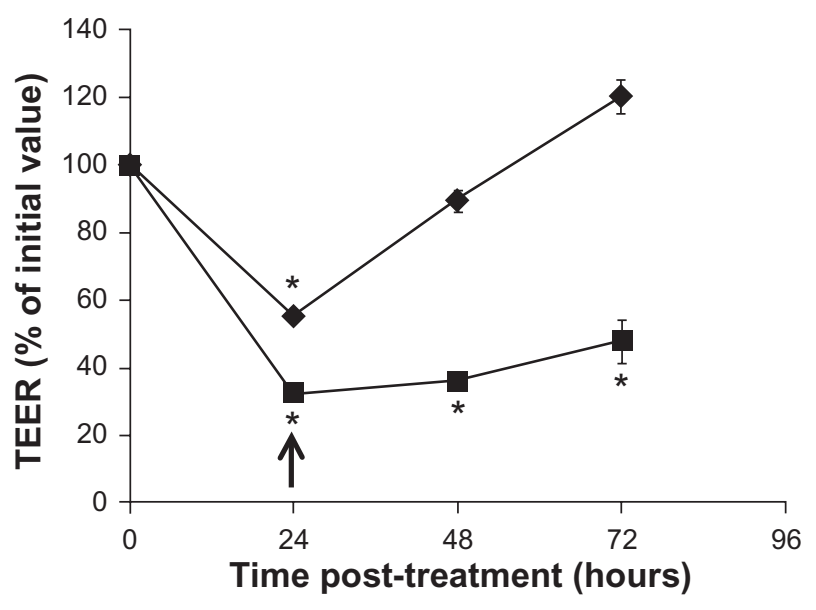

Figure 4 TEER over time upon removal of fSWCNT-COOH $(\bullet)$, or triton-x ( $\mathbf{\square})$. Caco- 2 cells were treated for 24 hours.

Notes: Arrow indicates the time of removal of the test agents and replacement of fresh medium. Asterisks represent statistical difference as compared to $\mathrm{t}=0$ with $P<0.05$. Abbreviations: TEER, transepithelial electrical resistance; fSWCNT-COOH, carboxylic acid functionalized single-walled carbon nanotubes. 


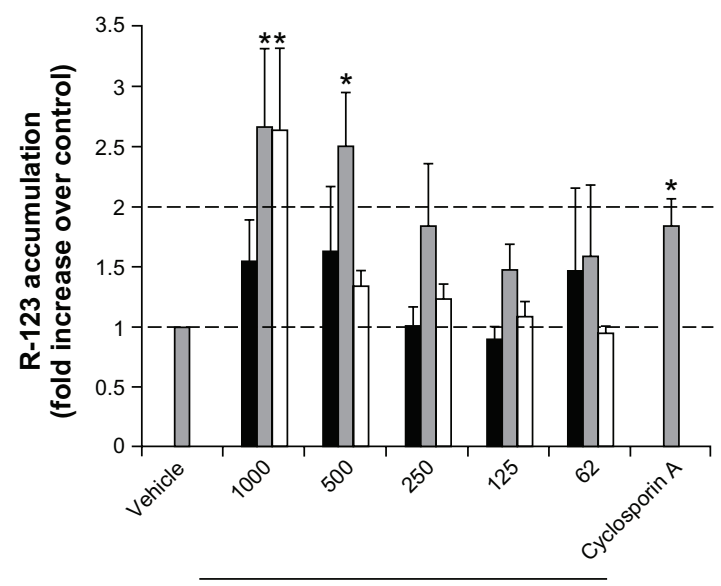

CNM $(\mu \mathrm{g} / \mathrm{mL})$

Figure 5 R-123 accumulation in Caco-2 cells after exposure to OH-fullerenes ( $\square$ ), fSWCNT-COOH ( $\square$ ), and fSWCNT-PEG ( $\square$ ).

Note: Asterisks represent statistical difference as compared to vehicle with $P<0.05$. Abbreviations: $\mathrm{OH}$-fullerenes, polyhydroxylated fullerenes; fSWCNT-COOH, carboxylic acid functionalized single-walled carbon nanotubes; fSWCNT-PEG, poly(ethylene glycol) functionalized single-walled carbon nanotubes; CNM, carbon nanomaterials.

the established P-gp inhibitor, cyclosporine A. Accumulation of the non P-gp substrate, rhodamine 110, ${ }^{33}$ in the presence of the three functionalized CNMs was not significantly different from that of vehicle control (data not shown). These results demonstrate that $f$ SWCNT-COOH and $f$ SWCNT-PEG have the ability to modulate P-gp efflux activity specifically, thus suggesting the potential in increasing oral bioavailability of P-gp drug substrates.

\section{Discussion}

Carbon nanomaterials have been extensively evaluated for biomedical applications such as drug delivery, with numerous proof-of-concept studies. ${ }^{3,4,7-9,14,15}$ However, very limited data are available to demonstrate their potential in oral drug delivery, partly because of the concern about the "fiber toxicities" of carbon nanotubes which are similar to those of asbestos. ${ }^{34}$ Animal toxicological studies involving oral dosing of CNMs, counting both functionalized and non-functionalized forms, are very limited. From the available studies, all except the one by Folkman et a ${ }^{35}$ reported minimal toxicity from oral dosing, as assessed by various endpoints including death, behavioral changes, genetic damages, embryo-fetal development, and maternal toxicity. ${ }^{16,19-21}$ In light of these previous findings, we were interested in characterizing the effect of these novel materials on the function of the intestinal epithelium and examining the potential of these CNMs in oral drug delivery applications, which has not been reported before.

Our results showed minimal cytotoxicity to Caco- 2 monolayer upon 24-hour exposure to the functionalized CNMs, and are in line with the animal studies reported by others that oral dosing of CNMs is not toxic. ${ }^{16,19-21}$ Of importance, C60-fullerenes and SWCNT, when administered at relatively high doses of $2 \mathrm{~g} / \mathrm{kg}$ and $1 \mathrm{~g} / \mathrm{kg}$, respectively, were excreted in feces 6 and 24 hours after oral administration, as demonstrated by the presence of compound-colored stool. ${ }^{16,21}$ Comparing with our results involving the recovery of TEER in the Caco-2 monolayer (Figure 4), the removal of $f$ SWCNT-COOH after 24 hours of exposure in our experiment agreed well with the time frame of fecal excretion of orally dosed SWCNT. Although a slight increase in cytotoxicity of $f$ SWCNT-PEG was observed after 72 hours of exposure, the LDH release induced by $f$ SWCNT-PEG treatment was comparable to other accepted PEG-based oral absorption modulators such as vitamin E-TPGS 1000 and Pluronic-P85. ${ }^{28,29}$ To support the application of CNMs in oral drug delivery, further studies could involve the determination of the amount of these CNMs that is absorbed orally and eliminated through fecal excretion. Compared to other in vitro studies that utilized Caco- 2 cells for cytotoxicity evaluation, our results are in line with those reported by Chiaretti et al in which no modification to Caco-2 cell growth was noted upon exposure to multi-walled carbon nanotubes. ${ }^{36} \mathrm{In}$ contrast, Jos et al reported Caco-2 plasma membrane damage from $f$ SWCNT-COOH exposure. ${ }^{37}$ This discrepancy is likely due to a much lower content $(<1 \%)$ of metal contaminants in our functionalized CNMs samples as compared to that used by Jos et al of $5 \%-8 \% \cdot{ }^{37}$ The removal of metal contaminants, known to induce cellular oxidative stress, has been shown to mitigate the cytotoxic effect of carbon nanotubes. ${ }^{38}$

It is interesting to note that the differences in morphologies and surface modifications of the three functionalized CNMs evaluated in our study did not give rise to a difference in Caco-2 cytotoxicity upon short-term (24 hour) exposure but rather a differential in cytotoxicity upon long-term (72 hour) exposure and their ability to modulate the barrier function of Caco-2 cell monolayer. Of importance, the effect of $f$ SWCNT-COOH on Caco-2 cells may be two-pronged: (1) increased paracellular drug transport via its reversible modulation on the tight junction, and (2) increased transport of P-gp substrates via inhibition of the P-gp efflux system. Such findings indicate considerable potential for $f$ SWCNT$\mathrm{COOH}$ as an oral drug delivery system. The selective effect of $f$ SWCNT-PEG on Caco-2 cells, whereby only P-gp efflux activity was inhibited without modulating tight junctions, would create unique opportunities for drug-specific oral delivery applications. Future studies involving the tracking of the intracellular fate of these novel CNMs would shed light on their differential biological properties. 
In summary, our current findings support the role of functionalized CNMs in drug delivery applications, and potentially open up doors to the delivery of a variety of drug molecules which may not be limited to those for cancer, infections, and neurodegenerative diseases that CNMs have been applied to as drug delivery systems. The challenges ahead in translating these novel materials into human applications include standardizing the methods for the production of these novel materials and for the characterization of their toxicological profiles. It is encouraging to note that various regulatory bodies such as US Food and Drug Administration are putting forward guidelines to help industry in the development of medical products that involve the use of nanotechnology.

\section{Acknowledgments}

The authors would like to thank Dr Giorgia Pastorin (Department of Pharmacy, National University of Singapore) for helpful discussions of this project. The research project is supported by Singapore Ministry of Education via the National University of Singapore Academic Research Fund.

\section{Disclosure}

The authors declare no conflicting interests in connection with this article.

\section{References}

1. Zhang Y, Bai Y, Yan B. Functionalized carbon nanotubes for potential medicinal applications. Drug Discov Today. 2010;15(11-12):428-435.

2. Ajayan PM, Zhou OZ. Applications of carbon nanotubes. In: Dresselhaus MS, Dresselhaus G, Avouris P, editors. Carbon Nanotubes: Synthesis, Structure, Properties, and Applications. Berlin, Germany: Springer-Verlag; 2001:391-425.

3. Partha R, Conyers JL. Biomedical applications of functionalized fullerene-based nanomaterials. Int J Nanomedicine. 2009;4:261-275.

4. Bianco A, Kostarelos K, Prato M. Applications of carbon nanotubes in drug delivery. Curr Opin Chem Biol. 2005;9(6):674-679.

5. Iijima S. Helical microtubules of graphitic carbon. Nature. 1991;354:56-58. Available at: http://www.nature.com/nature/journal/v354/n6348/ abs/354056a0.html.

6. Kroto HW, Heath JR, O’Brien SC, Curl RF, Smalley RE. C60: Buckministerfullerene. Nature. 1985;318:162-163. Available at: http://www. nature.com/nature/journal/v318/n6042/abs/318162a0.html.

7. Yang K, Wan J, Zhang S, Zhang Y, Lee ST, Liu Z. In vivo pharmacokinetics, long-term biodistribution, and toxicology of PEGylated graphene in mice. ACS Nano. 2011;5(1):516-522.

8. Yang K, Zhang S, Zhang G, Sun X, Lee ST, Liu Z. Graphene in mice: ultrahigh in vivo tumor uptake and efficient photothermal therapy. Nano Lett. 2010;10(9):3318-3323.

9. Feng L, Liu Z. Graphene in biomedicine: opportunities and challenges. Nanomedicine (Lond). 2011;6(2):317-324.

10. Patlolla A, Knighten B, Tchounwou P. Multi-walled carbon nanotubes induce cytotoxicity, genotoxicity and apoptosis in normal human dermal fibroblast cells. Ethn Dis. 2010;20(1 Suppl 1):S1-65-72.

11. Lindberg HK, Falck GC, Suhonen S, et al. Genotoxicity of nanomaterials: DNA damage and micronuclei induced by carbon nanotubes and graphite nanofibres in human bronchial epithelial cells in vitro. Toxicol Lett. 2009;186(3):166-173.
12. Fiorito S, Serafino A, Andreola F, Bernier P. Effects of fullerenes and single-wall carbon nanotubes on murine and human macrophages. Carbon. 2006;44(6):1100-1105.

13. Reddy AR, Reddy YN, Krishna DR, Himabindu V. Multi wall carbon nanotubes induce oxidative stress and cytotoxicity in human embryonic kidney (HEK293) cells. Toxicology. 2010;272(1-3):11-16.

14. Liu Z, Davis C, Cai W, He L, Chen X, Dai H. Circulation and long-term fate of functionalized, biocompatible single-walled carbon nanotubes in mice probed by Raman spectroscopy. Proc Natl Acad Sci U S A. 2008; 105(5):1410-1415.

15. Schipper ML, Nakayama-Ratchford N, Davis CR, et al. A pilot toxicology study of single-walled carbon nanotubes in a small sample of mice. Nat Nanotechnol. 2008;3(4):216-221.

16. Kolosnjaj-Tabi J, Hartman KB, Boudjemaa S, et al. In vivo behavior of large doses of ultrashort and full-length single-walled carbon nanotubes after oral and intraperitoneal administration to Swiss mice. ACS Nano. 2010;4(3):1481-1492.

17. Poland CA, Duffin R, Kinloch I, et al. Carbon nanotubes introduced into the abdominal cavity of mice show asbestos-like pathogenicity in a pilot study. Nat Nanotechnol. 2008;3(7):423-428.

18. Takagi A, Hirose A, Nishimura T, et al. Induction of mesothelioma in $\mathrm{p} 53+/-$ mouse by intraperitoneal application of multi-wall carbon nanotube. J Toxicol Sci. 2008;33(1):105-116.

19. Chen HHC, Yu C, Ueng TH, et al. Acute and subacute toxicity study of water-soluble polyalkylsulfonated C-60 in rats. Toxicol Pathol. 1998; 26(1):143-151.

20. Lim JH, Kim SH, Shin IS, et al. Maternal exposure to multi-wall carbon nanotubes does not induce embryo-fetal developmental toxicity in rats. Birth Defects Res B Dev Reprod Toxicol. 2011;92(1):69-76.

21. Mori T, Takada H, Ito S, Matsubayashi K, Miwa N, Sawaguchi T. Preclinical studies on safety of fullerene upon acute oral administration and evaluation for no mutagenesis. Toxicology. 2006;225(1):48-54.

22. Sayes CM, Fortner JD, Guo W, et al. The differential cytotoxicity of water-soluble fullerenes. Nano Lett. 2004;4(10):1881-1887.

23. Wilson G, Hassan IF, Dix CJ, et al. Transport and permeability properties of human Caco-2 Cells - an in vitro model of the intestinal epithelial-cell barrier. J Control Release. 1990;11(1-3):25-40.

24. Hidalgo IJ, Raub TJ, Borchardt RT. Characterization of the humancolon carcinoma cell-Line (Caco-2) as a model system for intestinal epithelial permeability. Gastroenterology. 1989;96(3):736-749.

25. Hilgers AR, Conradi RA, Burton PS. Caco-2 Cell monolayers as a model for drug transport across the intestinal mucosa. Pharmaceut Res. 1990; 7(9):902-910.

26. Belyanskaya L, Manser P, Spohn P, Bruinink A, Wick P. The reliability and limits of the MTT reduction assay for carbon nanotubes-cell interaction. Carbon. 2007;45(13):2643-2648.

27. Worle-Knirsch JM, Pulskamp K, Krug HF. Oops they did it again! Carbon nanotubes hoax scientists in viability assays. Nano Lett. 2006;6(6):1261-1268.

28. Zastre J, Jackson J, Bajwa M, Liggins R, Iqbal F, Burt H. Enhanced cellular accumulation of a P-glycoprotein substrate, rhodamine-123, by Caco-2 cells using low molecular weight methoxypolyethylene glycolblock-polycaprolactone diblock copolymers. Eur J Pharm Biopharm. 2002;54(3):299-309.

29. Collnot EM, Baldes C, Wempe MF, et al. Influence of vitamin E TPGS poly(ethylene glycol) chain length on apical efflux transporters in Caco-2 cell monolayers. J Control Release. 2006;111(1-2):35-40.

30. Anderson JM, Van Itallie CM. Physiology and function of the tight junction. Cold Spring Harb Perspect Biol. 2009;1(2):a002584.

31. Anderson JM, Van Itallie CM, Peterson MD, Stevenson BR, Carew EA, Mooseker MS. ZO-1 mRNA and protein expression during tight junction assembly in Caco-2 cells. J Cell Biol. 1989;109(3):1047-1056.

32. Schneeberger EE, Lynch RD. The tight junction: a multifunctional complex. Am J Physiol Cell Physiol. 2004;286(6):C1213-C1228.

33. Batrakova EV, Han HY, Alakhov V, Miller DW, Kabanov AV. Effects of pluronic block copolymers on drug absorption in Caco-2 cell monolayers. Pharm Res. 1998;15(6):850-855. 
34. Kayat J, Gajbhiye V, Tekade RK, Jain NK. Pulmonary toxicity of carbon nanotubes: a systematic report. Nanomedicine. 2011;7(1):40-49.

35. Folkmann JK, Risom L, Jacobsen NR, Wallin H, Loft S, Moller P. Oxidatively damaged DNA in rats exposed by oral gavage to C-60 fullerenes and single-walled carbon nanotubes. Environ Health Persp. 2009;117(5):703-708.

36. Chiaretti M, Mazzanti G, Bosco S, et al. Carbon nanotubes toxicology and effects on metabolism and immunological modification in vitro and in vivo. J Phys Condens Matter. 2008;20:474203.
37. Jos A, Pichardo S, Puerto M, Sanchez E, Grilo A, Camean AM. Cytotoxicity of carboxylic acid functionalized single wall carbon nanotubes on the human intestinal cell line Caco-2. Toxicol in Vitro. 2009;23(8):1491-1496.

38. Pastorin G, Nayak TR, Leow PC, Ee PLR, Arockiadoss T, Ramaprabhu S. Crucial parameters responsible for carbon nanotubes toxicity. Curr Nanosci. 2010;6(2):141-154. 


\section{Supplementary figures}

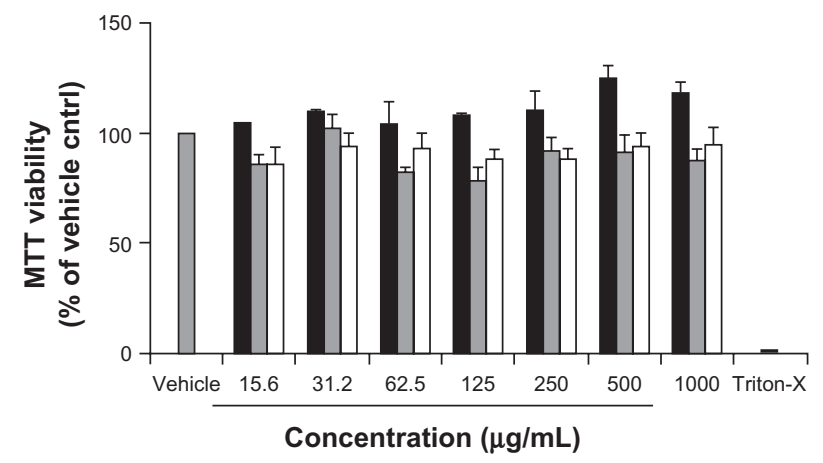

Figure SI Viability of OH-fullerenes (घ), fSWCNT-COOH ( $\square$ ), and fSWCNTPEG $(\square)$ as determined by MTT assay 24 hours post-treatment.

Abbreviations: $\mathrm{OH}$-fullerenes, polyhydroxylated fullerenes; fSWCNT-COOH, carboxylic acid functionalized single-walled carbon nanotubes; fSWCNT-PEG, poly(ethylene glycol) functionalized single-walled carbon nanotubes; MTT, 3-(4,5dimethyliazol-2-yl)-2,5-diphenyltetrazolium bromide.

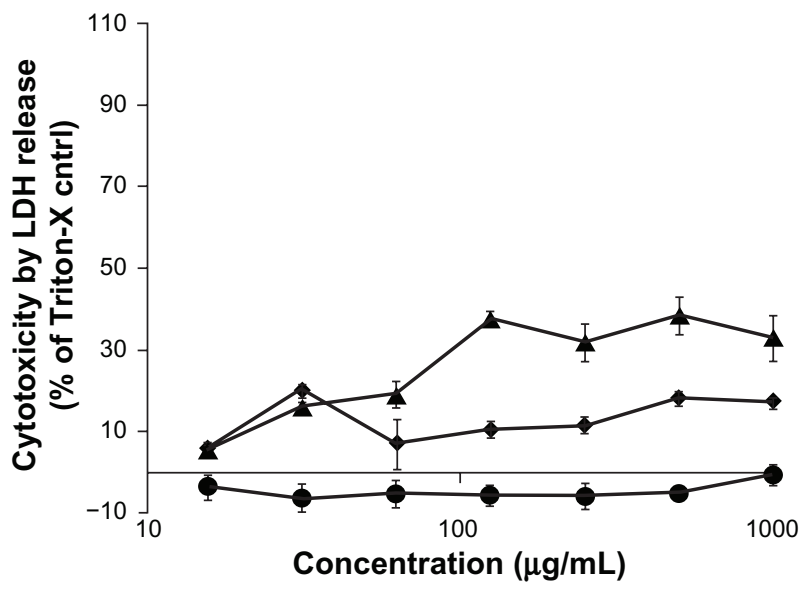

Figure S2 Cytotoxicity of $\mathrm{OH}$-fullerenes $(\bullet), f \mathrm{SWCNT}-\mathrm{COOH}(\boldsymbol{\bullet})$, and fSWCNT-PEG $(\mathbf{\Delta})$ after 72 hours of treatment.

Abbreviations: LDH, lactose dehydrogenase; $\mathrm{OH}$-fullerenes, polyhydroxylated fullerenes; fSWCNT-COOH, carboxylic acid functionalized single-walled carbon nanotubes; fSWCNT-PEG, poly(ethylene glycol) functionalized single-walled carbon nanotubes.

\section{Publish your work in this journal}

The International Journal of Nanomedicine is an international, peerreviewed journal focusing on the application of nanotechnology in diagnostics, therapeutics, and drug delivery systems throughou the biomedical field. This journal is indexed on PubMed Central, MedLine, CAS, SciSearch ${ }^{\circledR}$, Current Contents ${ }^{\circledR} /$ Clinical Medicine,
Journal Citation Reports/Science Edition, EMBase, Scopus and the Elsevier Bibliographic databases. The manuscript management system is completely online and includes a very quick and fair peer-review system, which is all easy to use. Visit http://www.dovepress.com/ testimonials.php to read real quotes from published authors 